\title{
Multi-step concept of the process of building a macromode
}

\author{
Maksadkhan Yakubov ${ }^{1 *}$, Gulchera Shadmanova ${ }^{2}$, Khabiba Karimova ${ }^{2}$, and Toshtemir \\ Khujakulov ${ }^{1}$ \\ ${ }^{1}$ Tashkent University of Information Technologies named after Muhammad Al-Khwarizmi, Tashkent, \\ Uzbekistan \\ ${ }^{2}$ Tashkent Institute of Irrigation and Agricultural Mechanization Engineers, Tashkent, Uzbekistan
}

\begin{abstract}
Building a macro and micro model of a multistage process is considered using the decomposition method, dividing the general technological system into interconnected control loops. A procedure for constructing an integrated macro model of a multistage process using a system of material balance equations for each control loop, a two-level mathematical model of a complex multistage system, developing principles for modeling and systematizing element models, the degree of aggregation, in general, controlling the technological properties of a material resource, calculating the schemes is reduced to solving a system of algebraic equations, modern technological scheme processing of a material resource, the procedure for constructing an integrated macro model multistage process (SME). A modern technological scheme for processing material resources cannot be carried out without returning circulating products when the product from subsequent nodes is sent for revision to the head of the technological scheme. The procedure for constructing an integrated macro model of SME $\mathrm{s}$ at two stages is considered. The task is to find the optimal solutions to the original mathematical model by interconnecting conditionally - optimal solutions of individual models. A comparison of various formal decomposition methods is performed according to previously determined criteria, and on this basis, the most preferable is chosen. When modeling multidimensional systems, one has to operate with various factors that have different units of measurement. Therefore, it is advisable to determine the dimensionless mathematical model since it is easily analyzed. The use of the latest mathematical modeling tools and modern methods of the flexible equation mainly determines the prospects of such studies.
\end{abstract}

\section{Introduction}

When creating SME s that allow to intensify processes and increase production efficiency, one of the most bottlenecks is the issue of modeling. At the same time, the structure and accuracy of a mathematical model largely determine the effectiveness of the entire management system of an SME. Often, models act as integrating elements of control

*Corresponding author: maksadhan@mail.ru 
systems, combining and processing information coming from various subsystems of control systems to obtain analytical and forecast data from the operation of the control object. The quality of such models - their adequacy, completeness, efficiency, largely determines the level and quality of control of the object as a whole.

Multistage processes are complex modeling systems, the analysis of the dynamics of operation and the synthesis of optimal solutions of which are possible in real situations only based on the use of computer technology. Therefore, the spectrum of requirements associated with the machine orientation of the models is presented to the simulation methods, with the possibility of using them in the tasks of a comprehensive analysis of the functioning of a multistage system and the synthesis of a flexible control system. In large terms, this part of the requirement is as follows: modularity, hierarchy, whether the form is a bridge, machine orientation, and versatility. This circumstance is due to the inherent features of mathematical modeling of SME s. The first group of discrete-continuous SME modeling features is related to the special quality of these systems, flexibility, the second to the high complexity of the modeling process, due to the large variety of elements and options for their integration, as well as the inclusion in consideration when modeling a large number of different types of elements whose coordination is a prerequisite for the organization of interaction elements. The third group of features is associated with the high responsibility and laboriousness of the SME modeling process.

The complexity of discrete-continuous SME s as objects of modeling is due to several objective factors, including the heterogeneity of the medium, the heterogeneity of the material on various grounds (size, shape, mineral composition of particles), the complexity of the interaction of various physic-chemical factors in the production process. As a result, each technological operation has a different degree of influence on the final results of the TP; therefore, in the SME management system, there are separate control loops of technological areas with a certain number of input and output material and information flows. The outlined contours can be viewed independently of the rest of the SME s, and it is necessary to develop control and identification algorithms for them $[1,2]$. For each office, its mathematical model is built, goals, management tasks, and technological constraints imposed on the management process are formulated. Building a unified mathematical model of a complex multistage production system as a whole is impossible because of the multi-factor, multi-criteria, and complexity of its operation. In these cases, it is necessary to decompose the modeled object into a finite number of subsystems, preserving the connections between the subsystems that ensure that the interaction of the subsystems is taken into account. The procedure for partitioning subsystems continues until such subsystems are obtained that, under the conditions of the problem under consideration, will be considered fairly simple and convenient for direct mathematical description, reflecting the properties of any one class of phenomena $[3,4]$. These subsystems, not subject to further dismemberment, are called elements of a complex system.

When choosing a mathematical apparatus that forms the basis for modeling, an important role is played by the characteristic properties of this class of systems, its elements, their interconnection, and interaction. A mathematical model of a complex multistage system consists of mathematical models of elements and a mathematical model of interaction between elements [5,6]. The complexity of building and using optimization models in managing SME s lies in the fact that, firstly, the introduction of optimization models will occur under the conditions of the current CU control systems. Secondly, the main processes of a multistage system are continuous-discrete and continuous, and third, non-linearity is characteristic of many of them [7,8]. Difficulties associated with the diversity of the composition of management tasks at individual levels and the need to consider them as a single whole with a variety of characteristics of elements and their modes of operation and the resulting hybrid integrated nature of mathematical models pose 
as priority issues of structuring a multistage system, developing principles for modeling and systematizing element models and the whole system. The definition of the optimal structure of the model is closely related to determining the periodicity of control, the frequency of correction of models of non-stationary subsystems of the complex, and the number of levels of the hierarchical system of flexible control. The hierarchical model is a set of system models built for all levels of SU SME s [9].

The system model structure is closely related to the degree of enlargement of technological areas as subsystems of the system. The degree of aggregation can serve as a quantitative measure of aggregation of aggregates - a quantity characterizing the number of system subsystems allocated during the construction of an integrated macro model. The degree of aggregation, in general, is represented by the ratio.

$$
S=k_{s} * m^{-1}
$$

Where $\mathrm{m}$ is the number of subsystems allocated when building an integrated macro model; $k_{s}$ - coefficient associated with the structural properties of the system [10].

The mathematical model of a multistage process is a set of operators (systems of equations) that reflect the models of individual local subsystems and circuits, i.e., the process model follows from the mathematical description of the technological operations involved in the process. Regardless of the nature of the technological scheme and the devices used, the type of material resource and its quality for each of its contour and the whole process, the law of material balance for the overall product and its components is valid. In [11] it is noted that to control the technological process of researching the properties of a material resource, it is necessary to know the qualitative and quantitative characteristics of each type of product obtained according to the technological scheme. Such a determination is made based on the balance calculation of the schemes, which is reduced to identifying the distribution of the material resource received in the process and its constituent components along the branches of the scheme. In mathematical terms, the balance calculation of schemes is reduced to solving a system of algebraic equations. For each circuit, you can make the following balance equations for the mass of the product [12, $13]$.

$$
\begin{aligned}
& \sum_{s=1}^{k_{j s}} \gamma_{i s}^{b x}=\sum_{s=k_{i 2+1}}^{k_{j s}} \gamma_{i s}^{v i x}, \\
& i=\overline{1, n} ; \\
& s=\overline{1, k_{i 1}}+\overline{k_{i 2}}
\end{aligned}
$$

and mass balance of ego components

$$
\sum_{s=1}^{k_{i 1}} \gamma_{i s}^{b x} d_{i j s}^{b x}=\sum_{\substack{s=k_{i 2+1} \\ j=1, m}}^{k_{i s}} \gamma_{i s}^{b i x} d_{i j s}^{b i x}
$$

Here $i$ - is the circuit contour number; $\mathrm{n}$ is the number of contours; $j$-is the component number; $\mathrm{m}$ is the number of components; $\mathrm{s}$ - contour product number; $k_{i 1} k_{i 2}$ - the number of products at the inlet and outlet of the circuit.

It should be noted that the solution of the system of balance equations is associated with several computational difficulties: a large dimensionality of the system, poor conditionality, uncertainty, incompatibility, and closeness of technological schemes for processing a 
material resource. A modern technological scheme for processing material resources cannot be carried out without returning circulating products when the product is sent from subsequent nodes to the head of the scheme for revision.

However, suppose at the balance calculation of the circuit contour, the task is reduced to determining the product yield by the model described by relatively simple one or multicomponent balance equations, then at the technological calculation of the scheme. In that case, it is necessary to calculate the full product characteristic, which requires the use of both balance and more complex equations. At the second stage, mathematical models are constructed for each selected contour, taking into account the tasks and limitations arising from the results of the first stage.

Consider the procedure for constructing an integrated macro model ICP [14].

For the $i$ - th circuit of the SME s, the material balance equations.

$$
\begin{aligned}
& \alpha_{i} \gamma_{\alpha_{i}}=\beta \gamma_{i}+\theta_{i} \gamma_{\theta_{i}} \\
& \gamma_{\alpha_{i}}=\gamma_{\beta_{i}}+\gamma_{\theta_{i}}
\end{aligned}
$$

where $\alpha_{i}, \beta_{i}, \theta_{i}$-is the content of a useful resource in nutrition, in the finished product and in dump products of the $i$ - th circuit; $\gamma_{\alpha_{i}}, \gamma_{\beta_{i}}, \gamma_{\theta_{i}}$ the mass of the material resource of the $i$ - th circuit, respectively, in the diet, in the finished product, and dump products. The control circuit of an SME can be represented as a multidimensional object (Fig. 1).

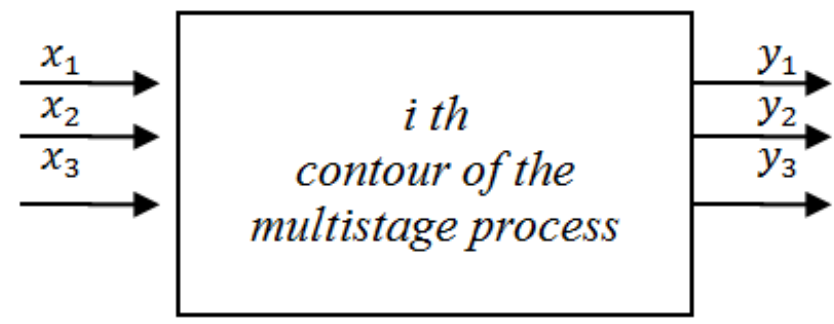

Fig.1. Block diagram of the control circuit

The task of building a mathematical model of a continuously functioning process is formulated as follows. Let the input parameter $x(t)$ with the $x_{1}(t)$, $x_{2}(t), \ldots \ldots, x_{\delta}(t)$ components affect $\tau$-the input $y(t)=y_{1}(t), \ldots, y_{r}(t)$ of the circuit and output the output parameter, with each of the output parameters, $y_{1}(t), y_{2}(t), y_{r}(t)$ completely determined in the probabilistic sense by all or often input $x_{1}(t), x_{2}(t), \ldots ., x_{\delta}(t)$.

\section{Methods}

Flows from other circuits and the initial flow of a material resource can flow into the power of the $i$ - th process loop. From here, we get expressions.

$$
\begin{gathered}
\alpha_{i}=\frac{\sum_{j=1}^{\pi}\left(\omega_{i j} \beta_{i} \gamma_{\beta_{i}}+N_{i j} \theta_{j} \gamma_{\theta_{j}}\right)+\alpha_{i}^{0} \gamma_{\alpha_{i}}^{0}}{\sum_{j=1}^{\mathrm{p}}\left(\omega_{i j} \beta_{i} \gamma_{\beta_{i}}+N_{i j} \gamma_{\theta_{j}}\right)+\gamma_{\alpha_{i}}^{0}} \\
\gamma_{\alpha_{i}}=\sum_{j=1}^{\pi}\left(\omega_{i j} \beta_{i} \gamma_{\beta_{i}}+N_{i j} \gamma_{\theta_{j}}\right)+\gamma_{\alpha_{i}}^{0},
\end{gathered}
$$

Where 


$$
\omega_{i j}=\left\{\begin{array}{l}
I, \text { if material resourse } \\
j-\text { go to the circuit input } \\
i-\text { go circuit, } \\
0, \text { otherwise, }
\end{array}\right\} ; N=\left\{\begin{array}{l}
I, \text { if material resourse } \\
j-\text { go circuit enter the } \\
\text { enterance } \\
i-\text { go circuit } \\
0, \text { otherwise }
\end{array}\right\}
$$

$\alpha_{i}^{0}$ is the content of the useful resource in the source stream; $\gamma_{\alpha_{i}}^{0}$ is output material resource in the original thread.

Changing and from $i$ to $n$ using equations (1), (2), we compose a system of linear algebraic equations; solving it, we find the following functions:

$$
\begin{aligned}
& \gamma_{\beta_{i}}=\gamma_{\beta_{i}}\left(\alpha_{1}^{0}, \alpha_{2}^{0} \ldots, \alpha_{n}^{0}, \gamma_{\alpha_{1}}^{0}, \gamma_{\alpha_{2}}^{0}, \ldots\right. \\
& \left.\gamma_{\alpha_{n}}^{0}, \quad \beta_{1}, \beta_{2}, \ldots, \quad \beta_{n}, \theta_{1}, i_{2}, \ldots, \theta_{n}\right), \\
& \gamma_{\theta_{i}}=\gamma_{\beta_{i}}\left(\alpha_{1}^{0}, \alpha_{2}^{0} \ldots, \alpha_{n}^{0}, \gamma_{\alpha_{1}}^{0}, \gamma_{\alpha_{2}}^{0}, \ldots\right. \\
& \left.\quad \ldots . \gamma_{\alpha_{n}}^{0}, \beta_{1}, \beta_{2}, \ldots, \beta_{n}, \theta_{1}, \theta_{2}, \ldots, \theta_{n}\right),
\end{aligned}
$$

Where $i=1,2, \mathrm{n}$.

If $\beta_{\pi}$ we $\gamma_{\beta_{\pi}}$ take for the content of a useful material resource in the finished product and for its output, then the system of equations is determined from the general material balance according to the technological scheme:

$$
\begin{gathered}
\sum_{i=1}^{\pi} \alpha_{i}^{0} \gamma_{\alpha_{i}}^{0}=\beta_{n} \gamma_{\mathrm{B}_{n}}+\theta_{o m b} \gamma_{\theta_{o m b}} \\
\sum_{i=1}^{\pi} \gamma_{\alpha_{i}}^{0}=\gamma_{\beta_{n}}+\gamma_{\theta_{o m b}}
\end{gathered}
$$

From this system, we find the expression, i.e., macro models, to maintain a useful material resource in dump products.

$$
\theta_{\text {omb }}=\beta_{\pi}-\frac{\beta_{\pi} \sum_{i=1}^{\mathrm{p}} \gamma_{\alpha_{i}}^{0}-\sum_{i=1}^{\pi} \alpha_{i}^{0} \gamma_{\alpha_{i}}^{0}}{\sum_{i=1}^{\pi} \gamma_{\alpha_{i}}^{0}-\gamma_{\beta_{n}}}
$$

Macro model regarding the content of a useful material resource in the finished product has the form.

$$
\beta_{\pi}=\theta_{o m b}-\frac{\theta_{o m b} g_{i_{o m b}}-\sum_{i=1}^{\mathrm{p}} b_{i}^{0} g_{\sigma_{i}}^{0}}{g_{v_{\mathrm{p}}}}
$$

Similarly, the macro model of the extraction of a useful product in the finished product is determined as follows:

$$
\mathrm{e}={ }^{v_{\mathrm{p}} g_{v_{\mathrm{p}}}} / \sum_{i-1}^{\mathrm{p}} b_{i}^{0} g_{b_{i}}^{0} .
$$

Thus, at the first stage of modeling, a macro model is built for the entire process, determined using only the input and output parameters of the contours. At the second stage, the micro model is constructed for individual local subsystems of the SME s following the principle given in $[15,16]$. 


\section{Results and Discussion}

The hierarchy of beginning systems under study is described using the original global mathematical model. Then, following the accepted composition of the hierarchy, the aggregated model of the central system and the detailed micro models of the local subsystems are separated from the original macro model. The task is to find the optimal solution to the original mathematical model by interconnecting conditionally - optimal solutions of individual models, then a comparison of various formal decomposition methods is performed according to previously determined criteria, and the preferable one is chosen on this basis [17]. In this case, the top-level model is built by aggregating the lower-level micro models following the conditionally optimal solutions previously obtained for them. In turn, the aggregated solution found using the top-level macro model is transmitted in the lower level models and used by them as a contour within which a detailed solution is searched. At the same time, it is impossible in principle to take into account all input parameters affecting the process and output variables; practically, it is necessary to confine ourselves to only a small part of the main determining input parameters; The task of the control system is to compensate for the effect of these disturbances. In addition, certain restrictions are imposed on the input and output parameters $g(x), g(y)$.

In the general case, the system of equations describing the process under study is expressed as follows:

$$
\begin{aligned}
& y_{j}=f_{j}\left(x_{1}, x_{2}, \ldots, \quad x_{l}, y_{1}, y_{2}, \ldots\right. \\
& \left.\ldots . y_{j-1}, y_{j+1}, \ldots y_{s}, a_{0}, a_{1}, \ldots, a_{m}\right) \\
& y=a_{0}+\sum_{i=1}^{k} a_{i} x_{i}+ \\
& +\sum_{l, i=1}^{k} a_{l i} x_{l} x_{i}+\sum_{i=1}^{k} a_{i i} x_{i}^{2}+\ldots \ldots
\end{aligned}
$$

The function $f_{j}(j=\overline{1, S})$ can be fairly well approximated by data functions $\overleftrightarrow{f}_{j}(j=$ $\left.\overline{1, S^{\prime}}, \quad S^{\prime}<S\right)$, up to a set of parameters, $b=\left(b_{0}, b_{1}, \ldots, b_{m}\right)$,i.e.

$$
\begin{aligned}
& y_{j} \approx y_{j}^{*}=\bar{f}_{j}\left(x_{1}, x_{2}, \ldots, \quad x_{l}, y_{1}, y_{2}, \ldots\right. \\
& \left.\ldots y_{j-1}, y_{j+1}, \ldots y_{s}, b_{0}, b_{1}, \ldots, b_{m}\right)
\end{aligned}
$$

or

$$
\begin{aligned}
& y_{j} \approx y^{*}=b_{0}+\sum_{i=1}^{k} b_{i} x_{i}+ \\
& +\sum_{\substack{l, i=1, l \neq i}}^{k} b_{l i} x_{l} x_{i}+\sum_{i=1}^{k} b_{i i} x_{i}^{2}+\ldots \ldots \ldots
\end{aligned}
$$

where $j=\overline{1, S^{\prime}}$ and $b=\left(b_{0,}, b_{1}, \ldots, b_{m}\right)$ are indefinite coefficients of the mathematical model.

Based on the experimentally obtained values $y_{j} \in g(y)$ and $x_{i_{д}} \in g(x)$,parameters $\left(j=\overline{1, S^{\prime}} ; \quad\right.$ д $\left.=\overline{1, S} ; \quad i=\overline{1, n}\right)$ (3), (4), estimates can be made using the method of least squares $[18,19]$. 
A block diagram of the construction of a mathematical model for a single subsystem of an SME is shown in Figure 2.

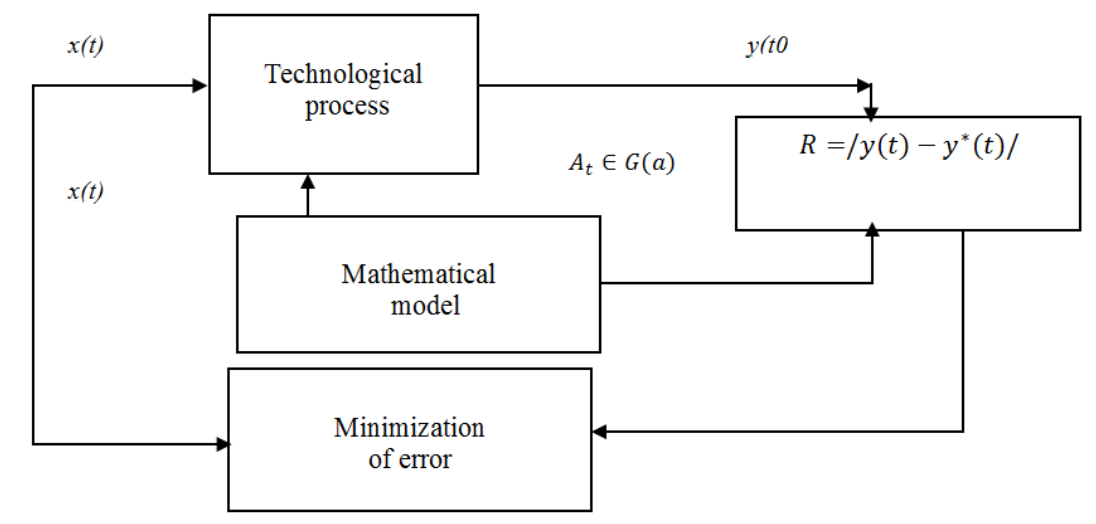

Fig. 2. Adaptation scheme of the mathematical model of SMEs.

When modeling multidimensional systems, one has to operate with various factors that have different units of measurement. Therefore, it is advisable to determine the dimensionless mathematical model since it is easily analyzed. You can also derive recurrence relations that allow you to move from a dimensionless model to a dimensional one. For this purpose, instead of components of the initial factors, standardized (dimensionless) components are introduced:

Where

$$
\begin{array}{r}
C_{x_{i s}}=\frac{x_{i s}-\mathrm{M}_{x i}}{A_{x_{i}}}, C_{y_{s}}=\frac{y_{s}-\mathrm{M}_{y}}{A_{y}}, \\
S=\overline{1, m ;} \quad i=\overline{1, n,}
\end{array}
$$

$$
\begin{gathered}
\mathrm{A}_{x_{i}}=\sqrt{\frac{\sum_{i=1}^{m}\left(x_{i s}-\mathrm{M}_{x i}\right)^{2}}{m-1}}, \\
\mathrm{Ay}_{y}=\sqrt{\frac{\sum_{s=1}^{m}\left(y_{s}-\mathrm{M}_{y}\right)^{2}}{m-1}}, \\
\mathrm{M}_{x_{i}}=\frac{1}{m} \sum_{s=1}^{m} x_{i s}, \\
\mathbf{M}_{y}=\frac{1}{m} \sum_{s=1}^{m} y_{s} .
\end{gathered}
$$

Here, the approximate regression equation is

$$
\begin{aligned}
& C_{y}=b_{1} c_{x 1}+b_{2} c_{x 2}+\ldots \\
& \ldots .+b_{n} c_{x n} .
\end{aligned}
$$

After simple transformations, we obtain a system of equations for the unknown coefficients, which in matrix form looks like this:

$$
D_{b}=\mathrm{v}
$$

Where 


$$
\begin{gathered}
D=\left|\begin{array}{l}
d_{11} d_{12} \ldots . . d_{1 n} \\
d_{21} d_{22} \ldots . . d_{2 n} \\
\ldots \ldots \ldots \ldots \ldots \ldots . . . \\
d_{n 1} d_{n 2} \ldots . . d_{n n}
\end{array}\right|, \\
b=\left|\begin{array}{l}
b_{1} \\
b_{2} \\
\ldots \ldots \\
b_{n}
\end{array}\right|, \quad v=\left|\begin{array}{l}
v_{1} \\
v_{2} \\
\ldots . \\
v_{n}
\end{array}\right| \text { or } D=d(i, j), \quad b=b(i), \quad v=v(i), \\
v_{j}=\frac{1}{m} \sum_{s=1}^{m} C_{y_{s}} C_{x_{j} s}, \quad i, j=\overline{1, n} \sum_{s=1}^{m} C_{x_{i} s} C_{x_{j} s}, \quad, \quad
\end{gathered}
$$

If for a quadratic matrix $\mathrm{D}$ there exists an inverse matrix, then, $D^{-1}$ multiplying (6) from the left $D^{-1}$ by, we find the unknown coefficients of the system $b_{i}$ :

$$
b=D^{-1} v
$$

Substituting the coefficients into an approximate regression equation (5), we find a dimensionless mathematical model (on a standardized scale) to describe the multidimensional system under study.

Thus, at present, mathematical modeling methods are the most convenient, reliable, and relatively cheap methods of researching real SME s. The use of the latest mathematical modeling tools and modern computer equipment mainly determines the prospects of such research.

Due to the presence of a greater number of heterogeneous disturbing influences, the SU SMEs will find it difficult to support the operation of the control object with the required quality and reliability. This problem is especially aggravated for multistage production processes with continuous-discrete and continuous nature, where the control cycle is limited from below by the speed, quality, and reliability of the measuring path, and from above by the speed, quality, and reliability of computers and communication channels.

Often, the direct determination of the values of process parameters in a production environment presents a certain difficulty. The complexity of solving this problem is because existing sensors and measuring devices do not have the required reliability, they are difficult to install on a functioning facility, and their cost is high.

To solve such problems, the following algorithm is proposed:

Let there are many technological parameters

$$
G=G\left\{x_{1}, x_{2}, \ldots, \quad x_{n}\right\}
$$

consisting of two subsets:

$$
G_{1}=G_{1}\left\{x_{1}^{(1)}, x_{2}^{(1)}, \ldots . x_{k}^{(1)}\right\}, \quad G_{1} \in G,
$$

whose values are easily definable, and

$$
G_{2}=G_{2}\left\{x_{1}^{(2)}, x_{2}^{(2)}, \ldots \ldots x_{k}^{(2)}\right\}, \quad G_{2} \in G,
$$

where values are difficult to determine.

In this case, the ratio 


$$
G_{1} \bigcap G_{2}=\Phi, \quad G=G_{1} \bigcup G_{2}
$$

and $k+s=n$.

If it is possible to determine the values $G_{2}$ of the parameters of the set, then it can be used to construct a system of models with respect to those parameters whose values are determined with a large time delay and costs.

In the operator form, the system of models can be represented as.

$$
G_{2}=t s\left(G_{1}\right)
$$

Where $\varphi$ is a functional operator selected from the arsenal of mathematical equations and methods that meet the requirements of specialists in solving specific problems, the system of models is stored in the PC memory, and at each control cycle, it is used to estimate and predict the values of difficult-to-determine parameters.

The essence of the proposed approach is to use the methodology of "flexible synthesis" [20-22], which consists of using information and its quality of organization at the stage of development and implementation in the procedures for developing control actions in control cycles at the stage of development and implementation of experimental data on the dynamics of the production process.

For a more detailed analysis of the proposed algorithm, we assume that there $x_{0}$ is a $(n \times m)$ matrix of experimental data with dimensions taken over a $\left(t_{0}, t_{1}\right)$ period of time, the elements $x_{i j}, i=\overline{1, n ;} \quad j=\overline{1, m}$; of which are input variables, including control variables and output variables. At the same time $j$ - means the number of the variable, $a$, and $u$ the number of measurements. Between these variables is the ratio.

$$
Y=F(x, u) \text {, }
$$

where $x$ - values of state variables among, $u$ - state of control variables, $Y$ - output values of state variables, $F$ - conversion operator.

We introduce a vector $\overrightarrow{x_{j}}=\left\{x_{1 j}, x_{2 j}, \ldots, x_{n j}\right\}$, that defines the set of measured values can be represented $\overline{x_{0}}$ as a set of columns

$$
\overrightarrow{x_{j}} j=\overline{1, m} \text {. }
$$

Based on the collected statistics of the experimental data $\overline{x_{0}}$, a correlation matrix is $\mathfrak{R}$ constructed, and the analysis is made according to the strength of the correlation connection (the relationship between the variables is weak, if - medium and - strong), the $0 \leq R_{i j} \leq$ 0.3 results of $\forall_{i} j=\overline{1, m}, \quad i \neq j$ which reduce the $0.3 \leq R_{i j} \leq 0.7$ dimension of the description of the production process to $\overrightarrow{x_{0}^{\prime}}$.

Thus, as a result of this procedure, $\overline{x_{j}}$ a subset is $R_{i j} \leq 0.7$ identified with the matrix $\overline{x_{0}}$ takes the form $\overrightarrow{x_{0}^{\prime}}$ of dimension $[n \times l],(l \leq m)$ and, by analogy with $(7)$, we have.

$$
Y=F_{1}\left(\overrightarrow{x^{1}}, U\right)
$$

Simultaneously with the above systematization of statistics of experimental data to improve the quality of operational management, at each step of the management cycle, the remaining population is classified $\overline{x_{j}}$ into $\overrightarrow{x_{0}^{\prime}}$ low-inertia (usually bistro-defined) $\overrightarrow{x_{j}^{\prime}}$ and strongly inertial variables, and to $\overrightarrow{x_{j}^{\prime \prime}}$ further reduce the dimensionality of the task of describing the state of the production process (determined) the relationship between them. 


$$
\begin{aligned}
\overrightarrow{x_{j}^{\prime \prime}} & =t s_{j-s}\left(\overrightarrow{x_{1}^{\prime}}, \overrightarrow{x_{2}^{\prime \prime}}, \ldots, \quad \overrightarrow{x_{s}^{\prime}}\right) \\
j & =\overrightarrow{s+1}, l .
\end{aligned}
$$

Systematization, in this case, is performed according to the following algorithm. Let $\overrightarrow{x_{0}^{\prime}}$ the vectors be grouped in a matrix $\overrightarrow{x_{j}^{\prime}}$ in such a way that at the beginning is located the first with vectors, $\overrightarrow{x_{j}^{\prime}}, j=\overline{s, 1}$ corresponding to low-inertia variables $\overrightarrow{x_{J}^{\prime \prime}}$, and then the vectors, corresponding to strongly inertial $j=\overline{s+1}, l$ variables in real time. As a result $\overrightarrow{x_{0}^{\prime}}$, the data matrix is represented in the form of two sub matrices $-\overrightarrow{x_{0}^{\prime \prime}}-$ - low-inertia and $-\overline{x_{0}^{\prime \prime \prime}}$ - strongly inertial.

When choosing the methodology of mathematical methods for determining the structure $ц_{j-s}, j=\overline{s+1}, l$, special attention will be paid to the method of the modeling function.

The criterion of the minimum of a quadratic functional of the form:

$$
R_{k p}=m_{E}+\sum_{i=1}^{n} \mathrm{o}_{i} k_{E}\left(\phi_{i}\right)
$$

where $k_{E}\left(\phi_{i}\right)$ is the value of the correlation function of the error at the moment $\phi_{i}, \mathrm{o}_{i}-$ some constant coefficients satisfying the condition $\sum_{i=1}^{n} \mathrm{o}_{1}^{2} \neq 0$. Then, the optimal estimate of the object operator can be searched for in the class of linear integral stationary operators

$$
Y(t)=A X(t)=\int_{-\infty}^{t} \operatorname{sh}(\phi) x(t-\phi) d \phi,
$$

where $щ(t)$ is the weight function of operator A.

Further, expressing the functional (10) through the original statistical data $(\operatorname{sh}(\phi)=0$ and $\phi<0, \quad \phi>t$ taking into account the physical reliability of the system (s), we obtain

$$
\begin{aligned}
& R_{k p}=m \int_{0}^{T} \operatorname{sh}(\phi) d t-m_{y}+ \\
& +\sum_{i=1}^{n} \mathrm{o}_{i}\left[\begin{array} { c } 
{ K _ { x } \cdot ( \phi _ { i } - l + } \\
{ \int _ { 0 } ^ { T } \int _ { 0 } ^ { T } + \phi ) \operatorname { s h } ( \phi ) d \phi d l - } \\
{ } \\
{ }
\end{array} \quad 2 \int _ { 0 } ^ { T } K _ { x y } \left(\phi_{i}+\right.\right. \\
&
\end{aligned}
$$

where, $m_{x}, m_{y}$ is the mathematical expectation of the input and output signals; $K_{x}(\cdot)$ и $K_{x y}(\cdot)$ - respectively auto and cross-correlation function of random processes $x(t)$ and $y(t)$. Expression (11) is a functional of the weight function of the type model (12).

$$
R_{k p}=\int_{0}^{T} \int_{0}^{T} \Phi[t, s, \operatorname{sh}(t), \operatorname{sh}(s)] d t d s .
$$

The integrand, in this case, has the form. 


$$
\begin{aligned}
& \Phi(\phi, l, \operatorname{sh}(t), \operatorname{sh}(l))= \\
& =\sum_{i=1}^{n} \mathrm{o}_{i} \mathrm{~K}_{x}\left(\phi_{i}-l+\phi\right) \operatorname{sh}(\phi) \operatorname{sh}(l)- \\
& -\frac{1}{\mathrm{~T}} \sum_{i=1}^{n} \mathrm{o}_{i}\left[\begin{array}{l}
\mathrm{K}_{x y}\left(\phi_{i}+\phi\right) \operatorname{sh}(\phi)+ \\
+\mathrm{K}_{x y}\left(\phi_{i}+l\right) \operatorname{sh}(\phi)
\end{array}\right]+ \\
& +\frac{m_{x}}{2 \mathrm{~T}}[\operatorname{sh}(\phi)+\operatorname{sh}(l)]
\end{aligned}
$$

and is a symmetric bilinear form with respect to $\operatorname{sh}(t)$ and $\operatorname{sh}(l)$.

It is easy to show that the estimate of the weight function of the object must satisfy the linear Fred Holm integral equation of the first kind.

$$
\begin{gathered}
\int_{0}^{\mathrm{T}} \sum_{i=1}^{n} \mathrm{o}_{i} \mathrm{~K}_{x}\left(\phi_{i}-l+\phi\right) s h_{0}(l) d l- \\
-\sum_{i=1}^{n} \mathrm{o}_{i} \mathrm{~K}_{x y}\left(\phi_{i}+\phi\right)+\frac{m_{x}}{2}=0
\end{gathered}
$$

where $\operatorname{sh}_{0}(l)$ is the optimal estimate of the weight function of the object by criterion (10). The solution of this equation can be found by the methods described in [7,23].

\section{Conclusions}

Considering the above estimates as some generalized coordinates $\overrightarrow{x_{0}^{\prime}}$, we obtain an effective mathematical description of complex production processes $\overrightarrow{x_{j}^{\prime}}$. The result of the above systematization allows them to be used to improve the efficiency and quality of the decision-making procedure in the ICP value information management cycles. The speed of the decision-making procedure in the control loop can be hung by further systematizing and ranking the elements of the submatrix $\overrightarrow{x_{0}^{\prime \prime}}$. For this purpose, the elements of the matrix $\overrightarrow{x_{0}^{\prime}}$ are divided into controllable and uncontrollable (but controllable) parameters with their further arrangement into sub-matrices of a lower dimension according to the following scheme.

We introduce $\mathrm{a} J_{1}=\{j\}$ set consisting of several uncontrollable variables. Following the above procedure, using the values of the correlation matrix $R_{i j}$, the ordering of the elements of the set is performed $J_{1}$. For this purpose, $\left(a_{0}, a_{d}\right)$ the interval based on technological considerations or the distribution law (normal, beta distribution, etc.) $d$ is divided into subintervals $\left[a_{0} a_{1}\right),\left[a_{1} a_{2}\right), \ldots\left[a_{d-1} a_{d}\right)$.

Further, the first subset is taken, $J_{1}$ for example, $I$ with an order number with the value of its possible values on the interval $\left[a_{0} a_{d}\right]$.

The procedure for $\overrightarrow{x_{k}^{\prime}}$ ordering in a subset $J_{1}$ is to group under the intervals and is determined by belonging to $\overrightarrow{x_{k}^{\prime}}$ the corresponding under the intervals. The whole set in each of the above sub-intervals form $\overrightarrow{x_{k}^{\prime}}$ correspondingly subsets $I_{1}, I_{2}, \ldots, I_{d}$. Following the selected sets, $I_{1}, I_{2}, \ldots, I_{d}$.the rows are rearranged in the matrix $\overrightarrow{x_{0}^{\prime}}$ in such a way that all vector values in the $\overrightarrow{x_{j}^{\prime}}$ sub-interval $\overrightarrow{x_{k}^{\prime}} \in\left[a_{0} a_{1}\right.$ )are at the beginning (at the top) of the matrix, then, etc $\overrightarrow{x_{k}^{\prime}} \in\left[a_{0} a_{1}\right)$. 
Thus, the initial data matrix $\overrightarrow{x_{k}^{\prime}}$ by the values of one of the elements $J_{2}$ of the subset is represented in the form of several submatrices of a significantly smaller dimension.

Next, for all rows with a sequence number, $i \in I_{\sigma}, b=\overline{1, d}$ the corresponding model (8) is constructed relative to some pre-selected output process indicator. For example, $q$ an empty variable with a sequence number, is an output indicator $\overrightarrow{x_{q}^{\prime}}$. Then model (8) takes the form

$$
\begin{aligned}
& \overrightarrow{x_{q_{1}}^{\prime}}=f_{1}\left(\overrightarrow{x_{1}^{\prime}}, \overrightarrow{x_{2}^{\prime}}, \ldots, \overrightarrow{x_{k}^{\prime}}, \ldots\right. \\
& , \overrightarrow{x_{q-1}^{\prime}}, \overrightarrow{x_{q+1}^{\prime}}, \ldots \\
& \left.. \overrightarrow{x_{s}^{\prime}}, \quad \overrightarrow{x_{s+1}^{\prime}}, \ldots \overrightarrow{x_{\ell}^{\prime \prime}}\right) \text { and } \overrightarrow{x_{k}^{\prime}} \in\left[a_{0} a_{1}\right), \\
& \quad \overrightarrow{x_{2}^{\prime}}=f_{2}\left(\overrightarrow{x_{1}^{\prime}}, \overrightarrow{x_{2}^{\prime}}, \ldots, \overrightarrow{x_{k}^{\prime}}, \ldots\right. \\
& . \overrightarrow{x_{q-1}^{\prime}}, \overrightarrow{x_{q+1}^{\prime}}, \ldots, \overrightarrow{x_{s}^{\prime}}, \overrightarrow{x_{s+1}^{\prime}}, . . \\
& \left.. \overrightarrow{x_{\ell}^{\prime \prime}}\right) \text { and } \overrightarrow{x_{k}^{\prime}} \in\left[a_{0} a_{1}\right), \\
& \quad \overrightarrow{x_{q_{d}}^{\prime}}=f_{d}\left(\overrightarrow{x_{1}^{\prime}}, \overrightarrow{x_{2}^{\prime}}, \ldots, \overrightarrow{x_{k}^{\prime}}, \ldots\right. \\
& , \overrightarrow{x_{q-1}^{\prime}}, \overrightarrow{x_{q+1}^{\prime}}, \ldots, \quad \overrightarrow{x_{s}^{\prime}}, \quad \overrightarrow{x_{s+1}^{\prime}}, . \\
& \left.\quad . \overrightarrow{x_{\ell}^{\prime \prime}}\right) \text { and } \overrightarrow{x_{k}^{\prime}} \in\left[a_{d-1} a_{d}\right) .
\end{aligned}
$$

An important advantage of the proposed approach to the organization of information conversion processing technology is that the constructed models of the form (10) can also be used to predict the characteristics of an output indicator of an object and to control them in the future, in actual production conditions.

The essence of the proposed algorithm for choosing the preferred process model with regard to technological situations is as follows. If the values of the variables of the object with the definition number $j, j=\overline{1, s}$ in the control cycle, $P$ then when they are substituted in (10), respectively, each model with different accuracy determines the values of the output indicator. In this case, the substitution of the values of these variables in the model of type (10) is carried out taking $\left(a_{r-1}, a_{r}\right)$.into account their affiliation to the interval. The error between the measured indicator value calculated by the model and the output indicator is determined as follows

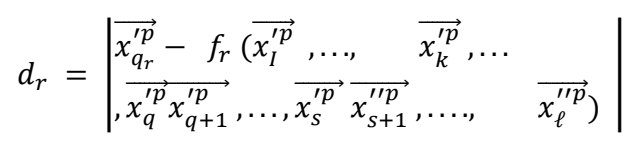

at $\overrightarrow{x_{k}^{\prime p}} \in\left(a_{r-1}, a_{r}\right)$, where $I \leq r \leq d$.

However, there may be cases when the chosen model with a satisfactory accuracy does not approximate the statistical dependence in the real process conditions. Leto $_{\mathrm{B}}$ be some given accuracy of the sought model. If $d_{r}<\mathrm{o}_{\mathrm{B}}$ that selected model can be used to solve subsequent optimization and control problems, $d_{r}>\mathrm{o}_{\mathrm{B}}$ otherwise the introduction of adaptation is necessary. One of the possible ways to improve the accuracy of models (9), (10) is to adjust them based on the accumulation of statistics during operation.

Thus, the practical implementation of all stages of the above methodology for selecting the most appropriate structure (type) of the process model can significantly reduce the PC memory and machine time spent on processing a large amount of information and increasing the model's reliability. Consequently, reliable models in the normal operation of 
the control object lead to a significant reduction in valuable material and energy resources and thereby improve the quality of management.

\section{References}

1. M.S.Yakubov. The concept of competitiveness and economic management system. International conference "Actual problems of development of info-communications and information society". Tashkent. pp.609-614. (2015)

2. Abdullaev A.A, Aliev R.A, Ulanov G.M, Principles of Industrial Automated Control Systems for Industrial Enterprises with Continuous Production. M.: Energy, p.440. (2013)

3. Aliyev E.M, Yakubov M.S. Programs for technological enrichment processes,Algorithms. (46) Tashkent: RICE of the Academy UzRes,. pp. 85-91. (2012)

4. Yakubov M.S. Organizational and functional structures of the automated control system for multistage production. Tashkent, Preprint. AN UzSSR NPO "Cybernetics", Z-8-20. Automated control systems of technological processes, pp. 28. (1989).

5. Yakubov M.S, Turgunov M.R. Creation of an integrated management system for freight traffic by rail. Bulletin of Tashkent State Technical University,pp. 86-90. (2017)

6. Yakubov M.S. Organizational and functional structures of the automated control system for multi- stage production. Tashkent. Preprint. AN UzSSR NPO "Cybernetics", Z-8-20. Automated control systems of technological processes, p.28. (1989).

7. Yakubov M.S, Azamov T.N. Features of the multistage process of firing ceramic bricks in a tunnel kiln. International Journal of Young Scientist. 10 (144), March 2017. (1). pp.111-114. (2017)

8. M.S. Yakubov, T.A. Khujakulov, M.M. Khusanov International Scientific and Technical Conference promising information technologies "The role of environmental assessment in the preparation and reconstruction of water sector projects" SAMARA, pp.1040-1044. (2017)

9. Polinov S.A. Recommendations for the selection of optimal directions for use and discharge modes of collector-drainage water from the Bukhara and Karshi water management regions. Report and research. Tashkent, SANIIRI,. p.129. (1989)

10. Shodmonova G, Islomov U, Inamov A, Samadov N. On one approach to numerical solution of nonlinear integro-differential equations. International Conference on Materials Physics, Building Structures and Technologies in Construction, Industrial and Production Engineering (MPCPE-2020). Vladimir, Russia, April 27-28, (2020).

11. Karimova X, Xikmatullaev S, Oymatov R. Vertical nonlinear oscillations of viscoelastic systems with multiple degrees of freedom. International Conference on Materials Physics, Building Structures and Technologies in Construction, Industrial and Production Engineering (MPCPE-2020). Vladimir, Russia, April 27-28, (2020).

12. Mirzaev S, Tukhtanazarov D, and Karimova Kh.Kh. Software for Determining Residual Oil Reserves in Oil Deposit Development. Construction Mechanics, Hydraulics and Water Resources Engineering (CONMECHYDRO - 2020). Tashkent Institute of Irrigation and Agricultural Mechanization Engineers. 23-25 April, (2020).

13. Mirzaev S, Kholmatova I, Shadmanova G, Yusupov M. and Kubyashev K.. Numerical modeling of two-dimensional two-phase filtration under frontal drive. Construction Mechanics, Hydraulics and Water Resources Engineering (CONMECHYDRO - 2020). Tashkent Institute of Irrigation and Agricultural Mechanization Engineers. 23-25 April, (2020). 
14. T.A Xujakulov. The problems of information and telecommunication technologies. The Republican Scientific and Technical Conference Tashkent. pp. 86-88 p. (2015)

15. M.S.Yakubov. The concept of competitiveness and economic management system. International conference "Actual problems of development of info-communications and information society". Tashkent. pp .609-614. (2015).

16. M.Yakubov, T.A.Xujakulov "Aral Sea" Materials for international scientific and practical conference from pp.188-190.

17. Shadmanova G, Karimova Kh.Kh. Information systems in water management. Information Science and Communications Technologies (ICISCT). (2019).

18. Khujaev I, Shadmanova G, Mamadaliyev X, Aminov X. The transition processes in gas pipe-lines. International Conference on Materials Physics, Building Structures and Technologies in Construction, Industrial and Production Engineering (MPCPE-2020). Vladimir, Russia, April 27-28, (2020).

19. Aynakulov Sh, Karimova X, Mamajonov M, Alibekov S, Shakirov B. Constructive device for sediment flushing from water acceptance structure. International Conference on Materials Physics, Building Structures and Technologies in Construction, Industrial and Production Engineering (MPCPE-2020). Vladimir, Russia, April 27-28, (2020).

20. Abdullayev Z.S. Modeling the Filtration of Groundwater In Multilayer Porous Media. International Conference on Materials Physics, Building Structures and Technologies in Construction, Industrial and Production Engineering (MPCPE-20), Vladimir, Russia. (2020).

21. Shadmanova G, Kodirov D, Tursunov O, Talipova D, Parpieva S, and Shafkarov B. System approach to renewable energy use in power supply. International Conference on Energetics, Civil and Agricultural Engineering (2020).

22. Kodirov D, Tursunov O, Karimova Kh, Akramova N, Parpieva S, and Shafkarov B. Application of hydro energy in small power supply systems. 1st International Conference on Energetics, Civil and Agricultural Engineering (2020).

23. M.S.Yakubov. The concept of competitiveness and economic management system. International conference "Actual problems of development of info-communications and information society". Tashkent. pp. 609-614 p.(2015) 